\title{
HIGH-SPEED OPTICAL DETECTORS IN YBCO FILMS WITH NON-GRANULAR AND FINE-GRAIN MORPHOLOGIES
}

\author{
Q. Tang, A. Driessen, L.H. Hilderink, R.P.J. IJsselsteijn, J. Flokstra and Th.J.A. Popma \\ Department of Applied Physics, University of Twente, P. O. Box 217 \\ 7500 AE Enschede, The Netherlands
}

\begin{abstract}
Optical detectors based on $\mathrm{YBa}_{2} \mathrm{Cu}_{3} \mathrm{O}_{7-\delta}$ thin films have been fabricated, which show a response time of a few ns. Typical dimensions of the active area are $10 \times 10 \mu \mathrm{m}^{2}$. No preamplifier is needed during operation. In this paper, we report on the optical response of detectors having nongranular and fine-grain morphologies to different pulsed laser radiation (e.g., a mode-locked Nd:YLF laser at $1.3 \mu \mathrm{m}$ and a GaAs diode laser at $0.9 \mu \mathrm{m}$ ). This study is focused on the detection sensitivity for measuring short optical pulses. It is found that the best NEP obtained with a fine-grain detector is on the order of $10^{-10} \mathrm{~W} / \mathrm{Hz}^{1 / 2}$, when irradiated by the $65 \mathrm{~ns}$ pulse-width diode laser.
\end{abstract}

\section{INTRODUCTION}

Much attention has been paid in recent years to the fast optical response of superconducting thin films to pulsed laser radiation [1-6]. Response times in the range of ns to several hundred fs were reported. From a practical point of view, the observed photoresponse can be used to develop a high-speed optical detector in either conventional or high- $\mathrm{T}_{\mathrm{c}}$ superconducting (HTS) thin films. The main advantage of such detectors is a broad response spectrum from the visible region to far-infrared (FIR) wavelengths. Therefore, it could provide in the future an alternative liquid-nitrogen cooled photodetector based on an $\mathrm{YBa}_{2} \mathrm{Cu}_{3} \mathrm{O}_{7-\delta}$ (YBCO) film for detecting very short (on the order of ns or less) optical pulses of radiation, for example, from free electron lasers [7].

The response time of HTS detectors at different wavelengths $(\lambda)$ has been examined by many authors. For example, Leung et al. [2] studied the performance of a granular YBCO detector and observed a nonbolometric effect in the optical response to pulsed FIR radiation at temperatures well below the transition temperature $\left(T_{c}\right)$. A response time of $20 \mathrm{~ns}$ was observed. Frenkel et al. [3] reported a nonbolometric optical response, as fast as $1 \mathrm{~ns}$, to a Nd:YAG $(\lambda=1.06 \mu \mathrm{m})$ laser radiation in non-granular YBCO films at $40 \mathrm{~K}$. Using a similar detector, Zheng et al. [4] observed a response time of less than 10 ns to a $\mathrm{CO}_{2}$ laser $(\lambda$ $=10.6 \mu \mathrm{m})$ at $10 \mathrm{~K}$. Furthermore, Carr et al. [5] reported a fast bolometric response (as fast as 4ns) of an YBCO detector to pulsed radiation from FIR to visible light sources. Somewhat discouragingly, however, the detectors with a high speed response always showed a very low responsivity (e.g., $0.01 \mathrm{~V} / \mathrm{W}[4]$ and $0.03 \mathrm{~V} / \mathrm{W}$ [3]), which is far lower than the requirements for practical applications.

In the following, we shall describe two kinds of detectors: a non-granular (NG) YBCO detector and a fine-grain (FG) one. We believe that with an FG morphology the detection sensitivity of HTS detectors can be improved. Possible mechanisms for the improvement in sensitivity can be the

Manuscript received August 24, 1992 following: (1) Films having fine and homogeneous grains can form a high density of boundary weak links. The boundary weak links may contribute coherently to the detected signal, but incoherently to the noise, so that the signal-to-noise $(\mathrm{S} / \mathrm{N})$ ratio will be high [1]. (2) It should be noted that, as in the case of $\mathrm{BaPbBiO}$ films, the reflection coefficient of polycrystalline films (including FG films) is smaller than that of single-crystal films [1]. Low reflectivity and high absorption will increase the detection efficiency.

\section{EXPERIMENTS}

The NG, c-axis oriented YBCO thin films, having a thickness of $70 \mathrm{~nm}$, were prepared by laser ablation onto $\mathrm{MgO}$ (100) or $\mathrm{SrTiO}_{3}(100)$ substrates. The deposition process has been described elsewhere [8]. The films had a zero-resistance $\mathrm{T}_{\mathrm{c}}$ of about $86-88 \mathrm{~K}$ and a critical-current density $\left(\mathrm{J}_{\mathrm{c}}\right)$ of $10^{5}$. $10^{6} \mathrm{~A} / \mathrm{cm}^{2}$ at $77 \mathrm{~K}$.
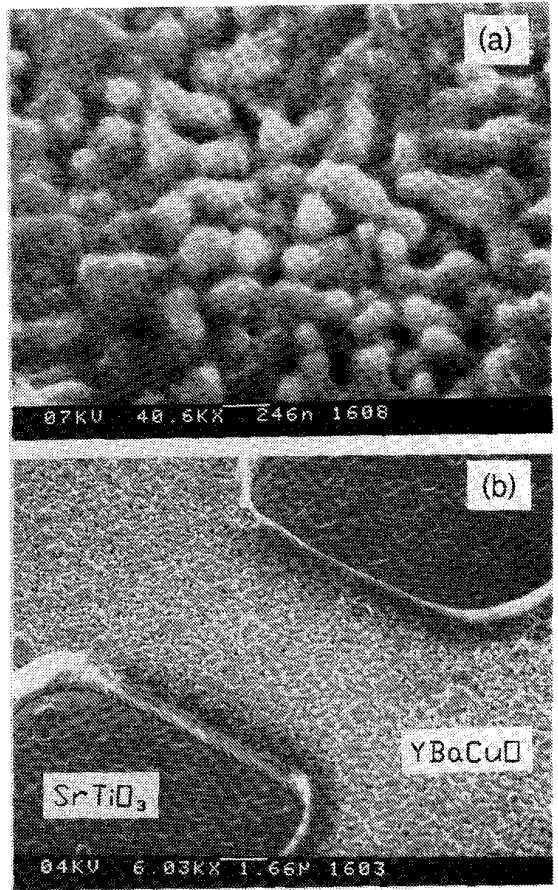

Fig.1 SEM photographs of (a) an FG film and (b) a bridgetype detector.

The FG YBCO thin films with $250 \mathrm{~nm}$ thickness on $\mathrm{SrTiO}_{3}(100)$ substrates were prepared by metalorganic aerosol deposition [9]. After deposition a post-annealing in oxygen was performed to produce a c-axis oriented film, which showed a good quality, $T_{c}=88-90 \mathrm{~K}$ and $\mathrm{J}_{\mathrm{c}} \geq 10^{4} \mathrm{~A} / \mathrm{cm}^{2}$ 
at $77 \mathrm{~K}$. Homogeneous grains with a diameter of about $200 \mathrm{~nm}$ have been obtained. FG morphology and a $10 \times 10 \mu \mathrm{m}^{2}$ bridge are shown in Fig.1. The temperature dependence of $J_{c}$ for the fine-grain film is shown in Fig.2, which is measured with the aid of the same patterned bridge. The solid line is a guide to the eye.

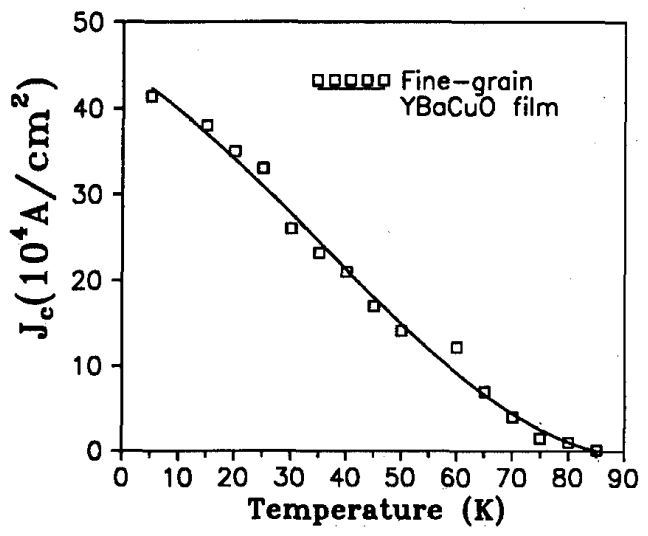

Fig.2 Critical-current density $\mathrm{J}_{\mathrm{c}}$ of a fine-grain YBCO film as a function of temperature.

The bridges were fabricated by standard photolithographic technique and $\mathrm{Ar}$ ion-beam milling. Afterwards oxygen plasma etching was performed to remove the photoresist. The active area used for this work is $10 \times 10 \mu \mathrm{m}^{2}$. The experimental setups of the measurements for $\mathrm{cw}$ and pulsed optical sources have been described earlier [10,11]. The optical response was obtained by using a dc bias current $\left(\mathrm{I}_{\mathrm{B}}\right)$ and measuring the change of voltage across the bridge. $A$ gold (or silver) layer with a thickness of $200 \mathrm{~nm}$ was evaporated onto YBCO films to make electrical contacts (resistance $<1 \Omega$ ). Several optical radiation sources were used: a mode-locked Nd:YLF laser with a pulse width of $75 \mathrm{ps}$ at a repetition rate of $77 \mathrm{MHz}(\lambda=1.31 \mu \mathrm{m})$, or a $\mathrm{GaAs}$ diode laser $(\lambda=0.9 \mu \mathrm{m}$, average power $1-3 \mathrm{~mW})$ with a pulse width of $65 \mathrm{~ns}$ at a repetition rate that can be varied from $10 \mathrm{kHz}$ to $30 \mathrm{kHz}$, or a cw HeNe laser $(\lambda=0.63 \mu \mathrm{m})$ which can be chopped with frequencies up to $4 \mathrm{kHz}$. An optical attenuator and a focus lens were placed at the front of the cryostat window. The laser beam was focused to a spot of approximately $30 \mu \mathrm{m}$ diameter. The average power (=peak power $\times$ pulse duty cycle) was measured with a calibrated powermeter (HP 8153A lightwave multimeter).

A fast, computerized sampling oscilloscope (Tektronix CSA803) with an input sampling head (Tektronix SD-26) having a rise time of about $25 \mathrm{ps}$ was used to analyse the response behaviour to the Nd:YLF laser. A standard $100 \mathrm{MHz}$ oscilloscope was used to display the response to the pulsed GaAs laser. The voltage noise of the detector was measured with a dynamic signal analyzer (HP 3561A).

\section{RESULTS}

\section{A. Optical response of NG detectors to the Nd:YLF laser}

The temperature dependence of the resistance $(R-T)$ for an NG film on a $\mathrm{MgO}$ substrate is shown in Fig.3. This measurement has been carried out with a three-probe geometry [11] and shows a finite background resistance which varies slightly with $I_{B}$. The inset of Fig. 3 shows the same curves in the temperature range between $35 \mathrm{~K}$ and $80 \mathrm{~K}$ with an expanded vertical scale in order to examine the details of the transition tails at 1 and $5 \mathrm{~mA}$. Our detectors are usually operated in this temperature range and with $I_{B}$ between 1 and $10 \mathrm{~mA}$. The dashed line with arrows below a certain temperature $T_{s}$ indicates the region where fast optical response is clearly observed. Here, "fast response" means that the voltage trace on an oscilloscope display can resolve the Nd:YLF laser pulses.

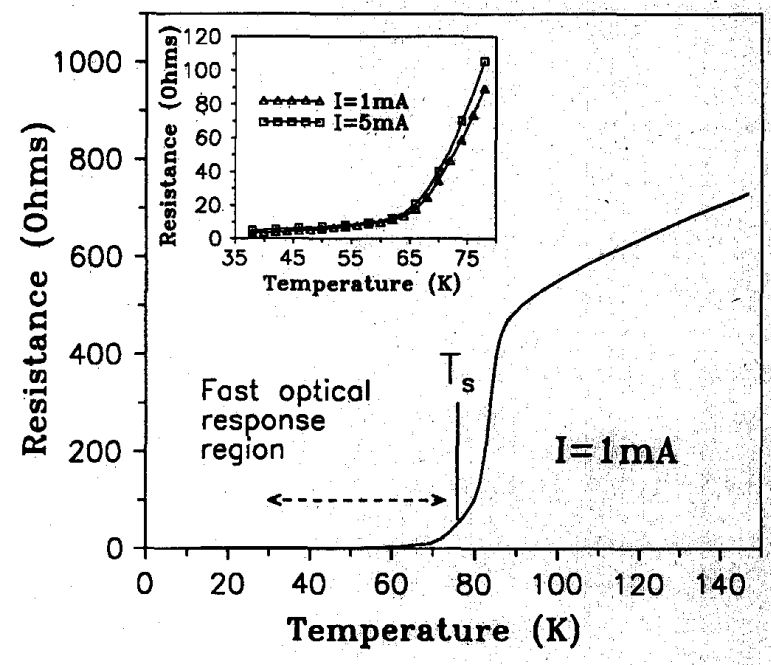

Fig.3R-T curve of an NG detector at $1 \mathrm{~mA}$. The inset shows the same curve with an expanded vertical scale.

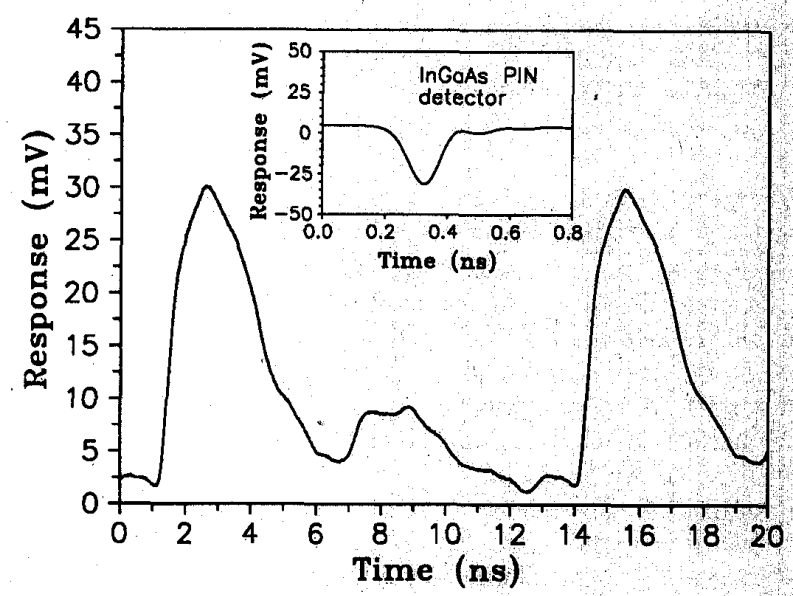

Fig. 4 Temporal optical response of an NG detector on MgO to the Nd:YLF laser measured at $66 \mathrm{~K}, 5 \mathrm{~mA}$ and $0.5 \mathrm{~mJ}$ per pulse. The inset shows the trace of the laser pulse detected by an InGaAs photodiode. 
A response curve at $66 \mathrm{~K}$ and $5 \mathrm{~mA}$ is presented in Fig. 4 . A $10 \%-90 \%$ rise time $\left(\tau_{\mathrm{r}}\right)$ of about $0.7 \mathrm{~ns}$ and a $90 \%-10 \%$ fall time $\left(\tau_{\mathrm{f}}\right)$ of about 2-3ns can be found. The inset of Fig.4 shows the Gaussian-shaped laser pulse detected by an InGaAs PIN diode (Antel AR-D25), which is an ultrafast photodetector $\left(\tau_{\mathrm{r}}<35 \mathrm{ps}\right.$, spectral response from 0.85 to $1.7 \mu \mathrm{m}$ [12]). The $\tau_{\mathrm{r}}$ of $0.7 \mathrm{~ns}$ and a full-width at half maximum (FWHM) of 2.5ns of our NG YBCO detector correspond to a $3-\mathrm{dB}$ cut-off frequency of $0.3 \mathrm{GHz}$. Note that the small peak between two consequent optical pulses is due to a transmission-line reflection in the circuit. This reflection can be eliminated by a well-designed device with improved electrical leads.

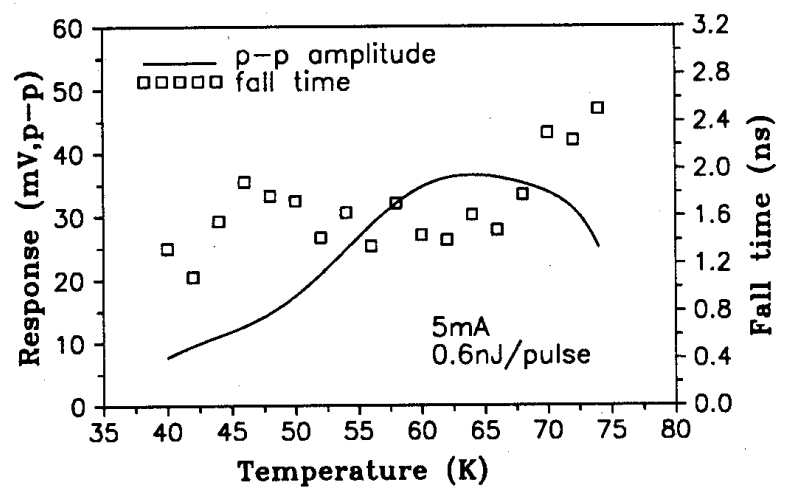

Fig.5 Response parameters $\tau_{\mathrm{f}}$ and the p-p amplitude as a function of temperature for an NG detector.

Fig.5 shows $\tau_{f}$ and the peak-to-peak (p-p) response amplitude as a function of temperature at $\mathrm{I}_{\mathrm{B}}=5 \mathrm{~mA}$. It can be seen that the $\tau_{f}$ decreases slightly from 75 to $60 \mathrm{~K}$, while the $p-p$ response amplitude changes significantly with temperature. Decreasing the temperature from 80 to $40 \mathrm{~K}$, the response amplitude first increases up to a maximum value, remains there constant for a relatively broad temperature range, and then decreases monotonously. If the temperature is further reduced, the response amplitude becomes too small to be detected due to our detection limit of $2 \mathrm{mV} / \mathrm{div}$ and noise of the system.

\section{B. Optical response of $F G$ detectors to $\mathrm{cw}$ lasers}

A peculiarity of the aerosol-deposited FG films is that, at temperatures well below $T_{c}$ and in a high current range, a Current Induced Voltage Step (CIVS) in the I-V curve has been found [13]. Related to this voltage jump, a resistive step in the R-T curve and a sharp optical response peak are observed. Fig. 6 shows the R-T curve and optical response of an FG detector for two different values of $\mathrm{I}_{\mathrm{B}}$ : $1 \mathrm{~mA}$ and $7.5 \mathrm{~mA}$. At $1 \mathrm{~mA}$ the $\mathrm{R}-\mathrm{T}$ curve shows a normal resistive transition. However, at $7.5 \mathrm{~mA}$, there is a significant shift to the low temperature direction. Finally, an abrupt resistive transition takes place. The optical responses at $1 \mathrm{~mA}$ and 7.5mA to a cw HeNe laser of $1 \mathrm{~mW}$ are shown in Fig.6(b).

In order to check that the sharp response peak in Fig.6(b) is not a processing-induced artifact, we have examined all of our aerosol-deposited FG films. The response behaviour is qualitatively the same. Furthermore, we have also examined
NG films. No sharp response peak could be found. Fig.7 shows the quasi-cw optical response to the GaAs laser as a function of $I_{B}$ at different temperatures. We chose temperatures from the midpoint of the transition to one low enough to observe the sharp response peak. Again it is shown that in the low $I_{B}$ range there is no sharp peak, while in the high $\mathrm{I}_{B}$ region, a sharp peak is present. Due to the presence of the sharp optical response peak, a high responsivity up to $10 \mathrm{~V} / \mathrm{W}$ can be obtained in the vicinity of the peak [11].

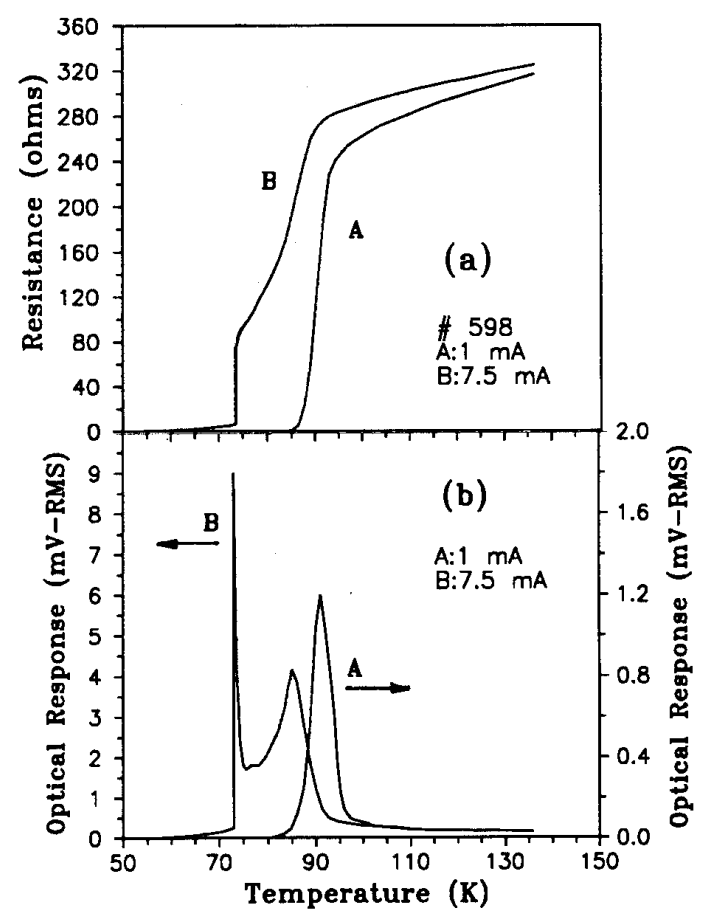

Fig.6 The resistance in (a) and cw optical response in (b) of an FG detector as a function of temperature.

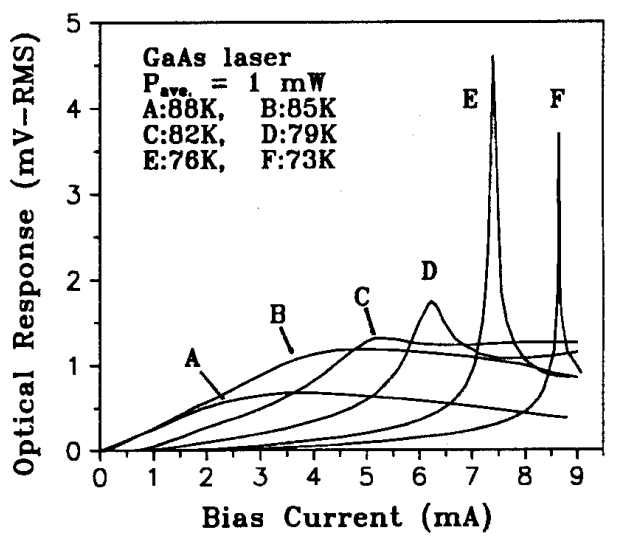

Fig.7 Optical response of an FG detector as a function of $I_{B}$ at different temperatures. 


\section{Optical response of $F G$ detectors to the pulsed $G a A s$ laser}

Figs.8(b) to (f) show the temporal optical responses of an FG detector to the 65ns-pulse-width GaAs laser at $5 \mathrm{~mA}$ and different temperatures from 85 to $65 \mathrm{~K}$, when the detection circuit is terminated with $50 \Omega$. Fig.8(a) is a reverse output trace of the InGaAs photodiode. In the set of traces in Fig.8, it can be found that the $\tau_{r}$ is almost the same. From high to low temperatures, the p-p response amplitude increases at the beginning and then decreases. The temperature dependence of the optical response in Fig. 8 is qualitatively in agreement with the results obtained with an NG detector in Fig.5.
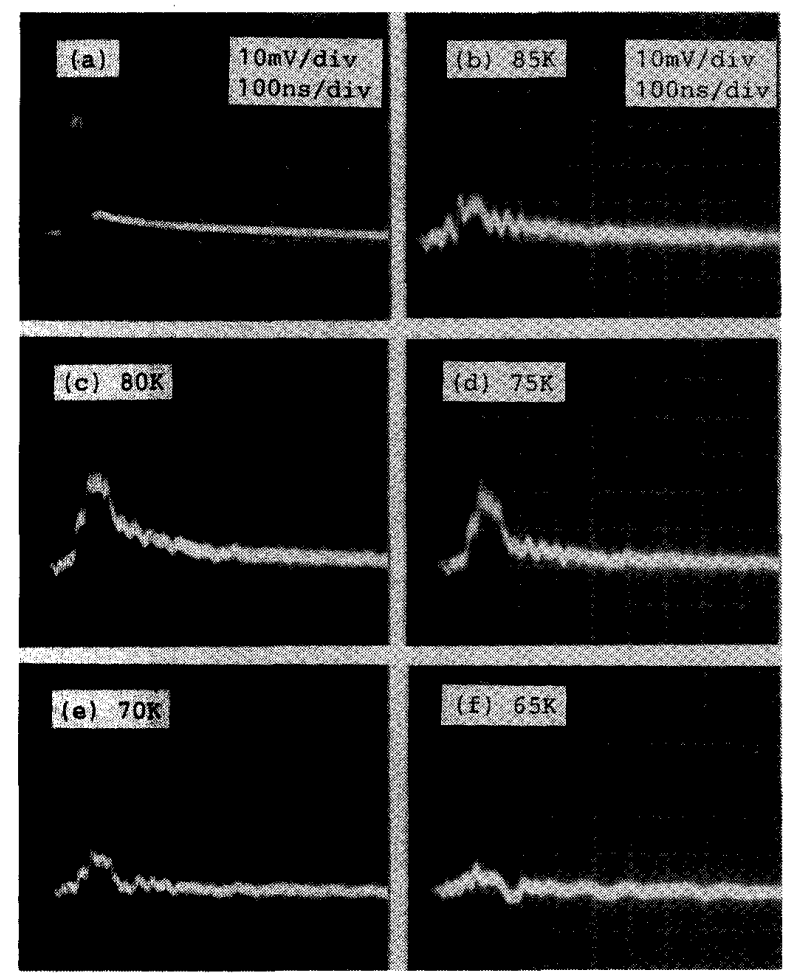

Fig.8 Oscilloscope traces of the GaAs laser, detected (a) by an InGaAs photodiode and (b)-(f) by an FG YBCO detector on $\mathrm{SrTiO}_{3}$.

In an approximation the FWHM response time, $\tau_{\mathrm{det}}$, of the FG detector can be obtained by:

$$
\tau_{\text {total }}^{2} \approx \tau_{\text {laser }}^{2}+\tau_{\text {det }}^{2} \text {. }
$$

where $\tau_{\text {total }}$ is the response time, as shown in Fig.8(d) $(\approx$ $80 \mathrm{~ns})$, and $\tau_{\text {laser }}$ is the laser's pulse width in Fig.8(a) ( $\left.\approx 65 \mathrm{~ns}\right)$. This results in $\tau_{\mathrm{det}} \approx 46 \mathrm{~ns}$.

\section{Detector noise and sensitivity}

The figures of merit of a photodetector for pulsed performance are not commonly defined in the literature. In the following, attempts have been made to estimate noise and a noise equivalent power (NEP) for an FG detector. The response curve to the pulsed GaAs laser at $75 \mathrm{~K}$ and $5 \mathrm{~mA}$, displayed on a dynamic signal analyzer, is shown in Fig.9. One clearly sees: (1) the optical response signals (peaks) in the Fourier transform spectrum, and (2) a voltage noise spectral density, $S_{v^{1 / 2}}(\mathrm{f})$. The $S_{v}{ }^{1 / 2}$ at $50 \mathrm{kHz}$ is $42 \mathrm{nV}^{\mathrm{H}} \mathrm{Hz}^{1 / 2}$.

Although the NEP can be simply defined as $S_{v}{ }^{1 / 2}(f)$ divided by a peak responsivity, in some cases, the results obtained by this way are not satisfactory. One of the reasons for that is the difficulty in the estimation of the absorbed peak power. In the following, we describe a comparative method for measuring the NEP for pulse performance. According to the definition, NEP is the incident power needed to obtain an $S / N$ ratio of 1 . If we use the same criterion ( $\mathrm{S} / \mathrm{N}=1)$, it is easy to measure the power required to generate such a signal output. This measurement can be carried out on YBCO detectors as well as on commercially available detectors under the same experimental conditions. If the NEP of the latter is known, then that of the former can be obtained. In our case, the InGaAs detector is taken as a reference, which has a NEP of about $5 \times 10^{-11} \mathrm{~W} / \mathrm{Hz}^{1 / 2}$ at 1.55 $\mu \mathrm{m}$ and about $1 \times 10^{-10} \mathrm{~W} / \mathrm{Hz}^{1 / 2}$ at $0.9 \mu \mathrm{m}[12]$.

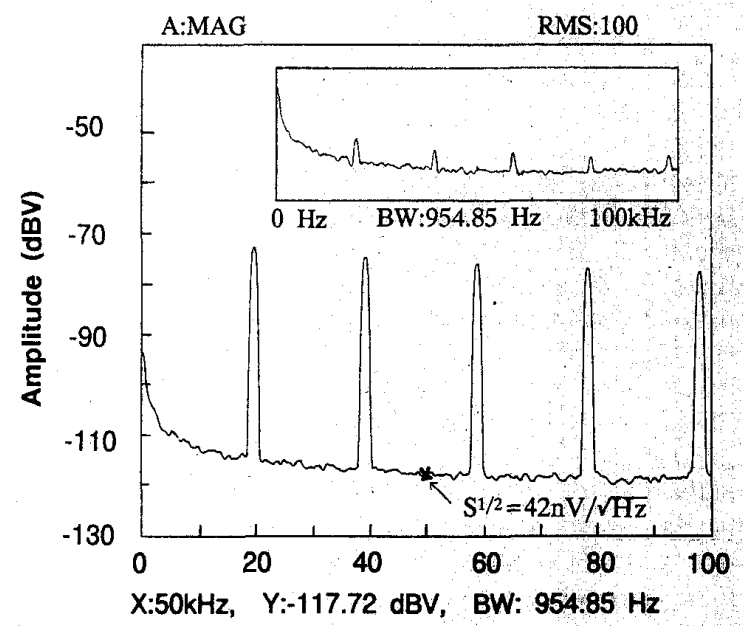

Frequency ( $\mathbf{k H z})$

Fig.9 Optical response of an FG detector to the pulsed GaAs laser. The inset shows a criterion for determining the relative NEP.

During the measurements, a neutral density filter is used to reduce the incident power. The response signal, as shown in the inset of Fig.9, is used as a criterion ( $S / N \approx 1$ ). It has been found that the incident optical power for an YBCO detector is about 8 times larger than that for the InGaAs detector, when the signal $(\mathrm{S} / \mathrm{N} \approx 1)$ can be yielded. Assuming the active area of both detectors is comparable (actually, the size of the YBCO detector is smaller), the NEP of the YBCO. detector can be roughly estimated to be $8 \times 10^{-10} \mathrm{~W} / \mathrm{Hz} / 2$ at $75 \mathrm{~K}$ and $5 \mathrm{~mA}$. The same analysis has been carried out for an NG detector on $\mathrm{SrTiO}_{3}$. The NEP obtained at $86 \mathrm{~K}$ and $5 \mathrm{~mA}$ is in the range of $(1-3) \times 10^{-9} \mathrm{~W} / \mathrm{Hz}^{1 / 2}$. 


\section{DISCUSSION AND CONCLUSIONS}

The mechanism of the fast optical response of HTS films at a temperature below $\mathrm{T}_{\mathrm{c}}$ is still a controversial issue whether it is a purely bolometric [5] or at least partially nonbolometric $[2,3,4]$ (e.g., breaking of the Cooper pairs and generation of quasiparticles) effect. We found that some of our results could not be properly explained by a simple heating effect. For example, we have estimated a lattice temperature rise ( $\delta T_{\text {pulse }}$ ) in the optical response to the $\mathrm{Nd}$ :YLF laser. Assuming a pure bolometric response signal: $\delta \mathrm{V}=\mathrm{I}_{\mathrm{B}} \times(\mathrm{dR} / \mathrm{dT}) \times \delta \mathrm{T}$, when $\delta \mathrm{V}, \mathrm{I}_{\mathrm{B}}$ and $\mathrm{dR} / \mathrm{dT}$ are known, the temperature rise $\delta \mathrm{T}$ (for a $1 \mathrm{~mW}$ optical power) near $\mathrm{T}_{\mathrm{c}}$ can be obtained. The $\delta \mathrm{T}$ of an NG device on $\mathrm{MgO}$ is about $5 \mathrm{mK} / \mathrm{mW}$. A $1 \mathrm{~nJ}$ energy pulse in a $2.5 \mathrm{~ns}$ time duration (see Fig.4) results in an average power of $400 \mathrm{~mW}$. Therefore, the $\delta \mathrm{T}_{\text {pulse }}$ (per pulse energy) in the same time duration can be calculated to be about $2 \mathrm{~K} / \mathrm{nJ}$. This value is in agreement with the results of a simple thermal model prediction giving a value of $1.5 \mathrm{~K} / \mathrm{nJ}$ [3]. This implies that the operating temperature of $66 \mathrm{~K}$ in Fig.4 is much lower than the temperature $\mathrm{T}_{\mathrm{c}}-\delta \mathrm{T}_{\text {pulse }}$. In other words, the photoinduced lattice temperature rise does not seem to be high enough to raise the device temperature above $T_{c}$.

On the other hand, in our experiments, some evidence can be provided to support a bolometric effect. Fig.4 shows that the FWHM response time $\tau$ of an YBCO detector on $\mathrm{MgO}$ is about $2.5 \mathrm{~ns}$, when the film thickness is comparable to the optical penetration depth $(\approx 70 \mathrm{~nm})$. The $\tau$ of a bolometer is equal to $\mathrm{C} / \mathrm{G}$, where $\mathrm{C}$ is the thermal capacity (data of $\mathrm{YBCO}$ films, see [5]) and $\mathrm{G}$ is the thermal conductance. Because the thermal conductivities of $\mathrm{SrTiO}_{3}$ and $\mathrm{MgO}$ are $0.2 \mathrm{~W} / \mathrm{cmK}$ [14] and $3.4 \mathrm{~W} / \mathrm{cmK}$ [15], respectively, it is easy to estimate that the $\tau$ on $\mathrm{SrTiO}_{3}$ is about $42 \mathrm{~ns}$ with the same geometry. This value is very close to the observed results from Eq.1.

Based on the above discussion, the optical response for both kinds of detectors could be mainly dominated by the heating effect of the radiation. The sharp optical response peak of an FG detector is associated with the CIVS, but the response mode does not change. The deviation between the observed results and a simple thermal analysis, as discussed above, is probably due to the complexity of the thermal processes, for example, thermal boundary resistance [16].

In conclusion, we have demonstrated YBCO detectors with NG and FG morphologies. The response time on the order of $n s$ and the NEPs of $10^{-9}-10^{-10} \mathrm{~W} / \mathrm{Hz}^{1 / 2}$ are observed, when irradiated with a pulsed GaAs laser at $0.9 \mu \mathrm{m}$.

\section{REFERENCES}

[1] Y.Enomoto and T.Murakami, "Optical detector using superconducting $\mathrm{BaPb}_{0.7} \mathrm{Bi}_{0.3} \mathrm{O}_{3}$ thin films", J.Appl. Phys., 59, 3807-3814, 1986.

[2] M.Leung, P.R.Broussard, J.H.Claassen, M.Osofsky, S.A.Wolf and U.Strom, "Optical detection in thin granular films of $\mathrm{Y}-\mathrm{Ba}-\mathrm{Cu}-\mathrm{O}$ at temperatures between 4.2 and 100K", Appl. Phys. Lett., 51, 2046-2047, 1987.

[3] A.Frenkel, M.A.Saifi, T.Venkatesan, P.England, X.D. $\mathrm{Wu}$ and A.Inam, "Optical response of nongranular high- $\mathrm{T}_{\mathrm{c}} \mathrm{Y}_{1} \mathrm{Ba}_{2} \mathrm{Cu}_{3} \mathrm{O}_{7-\mathrm{x}}$ superconducting thin films", J.Appl.Phys., 67, 3054-3068, 1990.
[4] J.P.Zheng, Q.Y.Ying and H.S.Kwok, "Y-Ba-Cu-O thin film infrared detectors", Physica C, 168, 322-326, 1990.

[5] G.L.Carr, M.Quijada, D.B.Tanner, C.J.Hirschmugl, G. P.Williams, S.Etemad, B.Dutta, F.DeRosa, A.Inam, T. Venkatesan and X.Xi, "Fast bolometric response by high $\mathrm{T}_{\mathrm{c}}$ detectors measured with subnanosecond synchrotron radiation”, Appl.Phys.Lett., 57, 2725-2727, 1990.

[6] S.G.Han, Z.V.Vardeny, K.S.Wong, O.G.Symko and G. Koren, "Femtosecond optical detection of quasiparticle dynamics in high- $\mathrm{T}_{c} \mathrm{YBa}_{2} \mathrm{Cu}_{3} \mathrm{O}_{7 . \delta}$ superconducting thin films", Phys.Rev.Lett., 65, 2708-2711, 1990.

[7] See, for example, M.F.Kimmitt, "Detectors for infrared free electron lasers", Infrared Phys., 32, 213-224, 1991.

[8] D.H.A.Blank, D.J.Adelerhof, J.Flokstra and H.Rogalla, "Preparation of $\mathrm{YBaCuO}$ thin films on various substrates by laser ablation", Physica C, 167, 423-432, 1990.

[9] Q.Tang, H.Albers, A.Driessen, L.T.H.Hilderink, P.V. Lambeck and Th.J.A.Popma, "Granular YBaCuO films prepared by metalorganic chemical aerosol deposition technology", J.Aerosol Sci., 21, Suppl. 1, S737-S740, 1990.

[10] Q.Tang, A.Driessen, P.Hoekstra, L.T.Hilderink, A.van Silfhout and Th.J.A.Popma, "Optical response of highly granular $\mathrm{YBaCuO}$ films prepared by non-vacuum aerosol deposition", J. Less-Common Metals, 164\&165, 1587-1594, 1990.

[11] Q.Tang, A.Driessen, L.H.Hilderink and Th.J.A.Popma, "CW and pulsed $\mathrm{IR}$ detection of aerosol deposited YBCO thin films", In: High $T_{c}$ Superconductor Thin Films, Proc. ICAM, Strasbourg, France, May 1991, pp.857-862.

[12] Antel AR-D25 Picosecond photodetector manual.

[13] A.Driessen, Q.Tang, P.Hoekstra and Th.J.A.Popma, "Dynamically enhanced supercurrents in granular superconducting Y-Ba-Cu-O thin films", J. Low Temp. Phys., 85, 347-357, 1991.

[14] E.F.Steigmeier, "Field effect on the Cochram modes in $\mathrm{SrTiO}_{3}$ and $\mathrm{KTaO}_{3}$ ", Phys. Rev., 168, 523-530, 1968.

[15] Q.Hu and P.L.Richards, "Design analysis of a high $\mathrm{T}_{\mathrm{c}}$ superconducting microbolometer", Appl.Phys.Lett., 55, 2444-2446, 1989.

[16] M.Nahum, S.Verghese, P.L.Richards and K.Char, "Thermal boundary resistance for $\mathrm{YBa}_{2} \mathrm{Cu}_{3} \mathrm{O}_{7-\delta}$ films", Appl. Phys.Lett., 59, 2034-2036, 1991. 